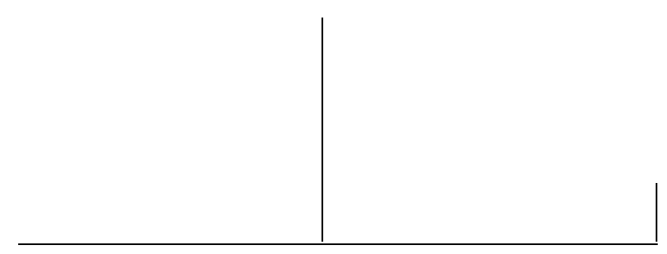

Rev. Latinoam. Psicopat. Fund., VI, 2, 89-98

\title{
L'analogie et le vrai en psychosomatique
}

\author{
Pascal-Henri Keller
}

Les théories en psychosomatique ont été élaborées dans la confrontation entre la psychanalyse et la médecine. Malgré les progrès de la médecine technoscientifique, les travaux actuels en psychanalyse, poursuivis dans le contexte de cette confrontation féconde, apportent régulièrement de nouveaux éléments de réflexion. La recherche présentée ici propose d'adjoindre à "l'instant de dire", notion développée par M.-J. Del Volgo, celle d' "analogisation”, fondée sur l'analyse de cette figure du discours qu'est l'analogie. La présentation du cas d'une patiente atteinte d'eczéma permettra de donner toute sa consistance à cette notion, dont les effets peuvent, à terme, renouveler la vision "défectologique" propre au courant dit "psychosomatique".

Mots clés: Psychosomatique, psychanalyse, analogie, eczéma 
Lorsque la maladie organique fait irruption, ce qu'elle déclenche en tant que phénomène indésirable, ce sont des actes destinés en priorité à le faire cesser, et secondairement à en empêcher le retour. C'est ainsi que les actes se succèdent aux actes, mêlant au point de les confondre, les actions exercées sur le corps du malade, et les actes de parole. Si l'avantage revient incontestablement et depuis longtemps aux gestes concrets en quête d'efficacité, les actes de parole n'en n'ont pas moins été associés à leur réalisation, le plus souvent dans une visée symbolique d'essence religieuse. Mais dans nos sociétés occidentales où la montée en puissance de la médecine techno scientifique impose le recours à un perfectionnement toujours plus grand de ces actions concrètes, on constate qu'ils sont loin de s'être totalement substitués aux actes de parole. Ceux-ci, dégagés peu à peu de l'emprise religieuse, parviennent à s'imposer à travers les attentes des patients et de leurs familles.

Ce que l'on désignera ici par "acte de parole" correspond à tout engagement dans un échange parlé avec une personne malade, indépendamment de la perspective d'une intervention sur le corps réel de cette personne. C'est de la psychanalyse que l'acte de parole, accompli dans ces conditions, a reçu ses titres de noblesse, avant toutefois d'être mêlé aux théories en psychosomatique; à terme, l'ambition de ces théories était en effet, schématiquement, d'autoriser une action plus efficace sur la pathologie frappant le corps malade. Ces théories ne parvenant pas à s'imposer sur ces bases dans la communauté scientifique (Keller, 2000), il a fallu repenser la nature même de l'acte de parole, tel qu'il s'accompli dans le cadre médical. Médecin et psychanalyste, M.-J. Del Volgo désigne son travail auprès des personnes malades comme "l'instant de dire"; elle définit également l'enjeu de ce travail comme ce qui autorise à "rétablir dans ses droits le mythe et le roman du malade (...), (à dire) le vrai de la maladie du malade, c'est à dire du sens et de la fonction intersubjective qu'elle prend dans son dialogue avec le médecin" (1997, p. 40). Dans ce contexte de surgissement d'une maladie, la dimension dialogale inhérente à la situation 


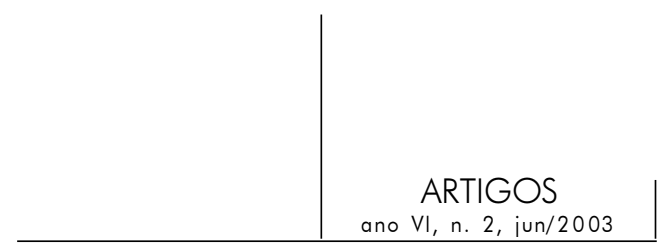

clinique ouvre des perspectives nombreuses, non seulement à la psychologie, mais également à l'anthropologie (Durif-Bruckert, 1994; Lebreton, 1985; Laplantine, 1986), à la sociologie (Herzlich \& Pierret, 1990), ou bien sûr à la psychanalyse (Keller, 2002).

D’une façon générale, la consultation des principales recherches entreprises dans ce domaine par la psychanalyse,${ }^{1}$ la psychosociologie (Herzlich \& Pierret, 1990), la psychologie clinique (Gori et Del Volgo, 1991) ou la psychologie de la santé (Bruchon-Schweitzer \& Dantzer, 1994), donne un aperçu de l'intérêt que suscite dans l'ensemble des sciences humaines la participation subjective de la personne malade aux soins qu'elle sollicite. Malgré leur diversité, toutes ces approches se rejoignent cependant sur une notion centrale, selon laquelle l'histoire individuelle d'une personne malade joue un rôle considérable dans la "coloration" affective de son engagement thérapeutique; ce que pourrait résumer la formule de Françoise Amiel-Lebigre (1993): “...le travail du fantasme organise de manière singulière l'univers représentationnel et affectif. C'est à partir de son histoire personnelle (et) de certains événements (...) que la personne interprète sa maladie et mobilise ses ressources personnelles et sociales" (Amiel-Lebigre et GognalonsNicolet, 1993). A partir de là, chacune des disciplines concernées s'emploie bien entendu à aborder le problème sous l'angle qui correspond le mieux à ses propres paradigmes (Keller, 2000, p. 172-190).

A sa façon, en insistant sur l'hétérogénéité des discours biologique et psychanalytique, Pierre Fédida (1994) contribue à cette tentative de réhabilitation du discours du patient, non pas dans le but d'opposer sa pertinence à celle du discours médical, mais bien dans celui de restituer à chacun sa légitimité propre. Les conséquences de cette tentative d'éclaircissement comportent, entre autres questions, celle que Fédida formule en termes de "métaphoricité de la plainte somatique dans la situation analytique"; ce qui l'amène logiquement à rappeler que "les contenus somatiques d'un patient affecté d'anomalies organiques ne sauraient être traitées dans la cure autrement que comme du matériau psychologique" (Ibid., p. 131).

Accepter de se saisir de cette hétérogénéité des discours implique tout à la fois d'adopter à leur égard une attitude psychosémiotique éclairée, et de restituer dans le même temps à chacun de ces discours sa logique propre: logique du sujet d'un côté, logique biologique de l'autre (Ducousso-Lacaze. et Keller, 1995, p. 615-40). Autant dire que cet effort de distanciation ne va pas de soi, impliquant même une rigueur accrue, à l'instar du traducteur d'un texte important écrit dans une autre langue que la sienne, soucieux d'être compris par son lecteur tout autant

1. Représenté essentiellement par le courant dits "psychosomatiques", cf. P-H. Keller, La médicine pasychosomatique en question ou Le savoir du malade. 


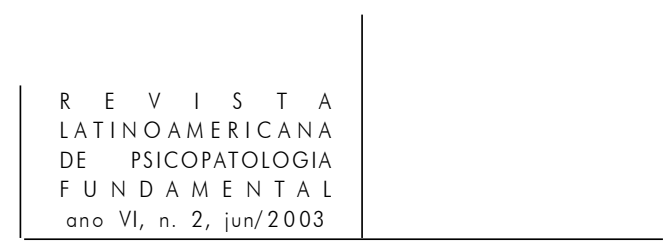

que respectueux de l'intention de l'auteur du texte original. Dans son célèbre texte de 1929, Ferenczi (1932) propose de rapprocher l'incompréhension qui s'installe entre enfant et adulte de l'incompréhension qui existe entre personnes ne parlant pas la même langue.

Dans le raisonnement qui découle de cette proposition, la confusion des langues déclenche des passages à l'acte de la part de l'adulte, justifiés de son point de vue, mais traumatisants pour l'enfant. Le premier parle la langue de la passion (et de la sexualité génitale), le second parle la langue de la tendresse (fantasmes ludiques et érotiques). Quant aux conséquences de ces "voies de faits" infligées à l'enfant, Ferenczi affirme qu'elles sont nombreuses et se prolongent à l'âge adulte. Cette analogie, transposée dans le domaine de la maladie somatique traitée par la médecine, revient à dire ceci: de même que, dans leur rencontre, l'adulte abuseur ne parle pas la même langue que l'enfant, le médecin qui reçoit un nouveau malade, ne parle pas non plus la même langue que son nouvel interlocuteur. Ou en termes plus classiques (Secrétan, 1984): du point de vue de la langue avec laquelle ils se parlent, le patient est au médecin ce que l'enfant est à l'adulte abuseur. On pourrait dire plus précisément concernant la langue, que celle (profane) dans laquelle le patient s'exprime est celle de la désignation subjective de son mal, alors que celle (savante) dans laquelle le médecin lui répond est celle de la guérison objective et raisonnée.

Le raisonnement analogique consiste dans ces conditions à réfléchir ainsi: de même que l'enfant qui s'adresse à l'adulte lui parle la langue de la tendresse, de même le malade qui s'adresse au médecin lui parle la langue de la subjectivité. En ce qui concerne la réponse qui leur est faite, à l'un comme à l'autre, tandis que l'adulte abuseur répond à l'enfant dans la langue de la passion, le médecin répond au malade dans la langue de l'objectivité scientifique à visée curative. Un dernier point concernant cette analogie et permettant d'en prendre la véritable mesure. Dans la situation dont parle Ferenczi (soumission-fascination de l'enfant pour l'adulte), l'attitude la plus "responsable" consiste, selon lui, à faire en sorte que l'enfant se "déprenne" de cette identification, qui, sans cela, finit par ressembler à un véritable assujettissement. Grâce à l'analogie établie avec la relation médecin/malade, cette recommandation permet de mettre davantage encore l'accent sur l'attitude subjective du médecin, tant est fréquente ou considérée comme rationnelle, l'ambition chez nombre de certains médecins de transformer en alter ego leur interlocuteur malade. La contrainte dans laquelle sont placés certains malades, d'avoir à parler la langue médicale, objectivante, au détriment de la leur, subjectivante, n'a pas, loin s'en faut, les même conséquences que celle qui pèse sur les enfants abusés; dans la plupart des cas au contraire - et nous atteignons là les limites du raisonnement analogique - elle permet au patient d'accélérer le rétablissement de sa santé. Toutefois, on peut 


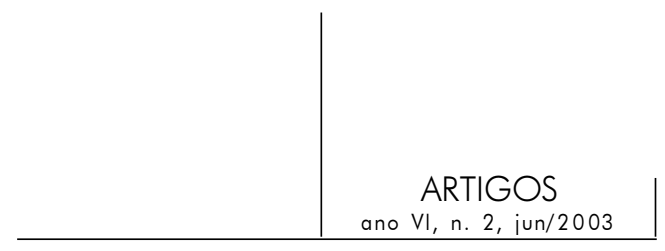

également concevoir que certains patients acceptent de guérir comme malgré eux, sans avoir véritablement réussi à transposer dans leur langue, le désir des médecins de les débarrasser de leur maladie.

Comme on vient de voir, l'acte de parole, fondateur de la relation qui s'établit entre une personne malade et son interlocuteur, impose pour chacun des deux partenaires en présence, de signifier l'enjeu de sa rencontre avec l'autre. A ce titre, si le médecin praticien est tenu d'indiquer au malade le contenu de ses intentions thérapeutiques, l'interlocuteur psychologue ou psychanalyste est $a$ contrario tenu de s'en démarquer. A moins de vouloir perpétuer indéfiniment les propositions aporétiques de la théorisation psychosomatique en cédant à sa logique causaliste, il revient aujourd'hui aux cliniciens chercheurs de faire de nouvelles propositions concernant l'enjeu spécifique de leur intervention. Dans la dynamique de ce que M.-J. Del Volgo nomme "l'instant de dire" (cf. plus haut), je propose de caractériser l'un de ses aspects en termes d' "analogisation" du discours; cette proposition visant in fine, à remplacer la perspective "défectologique" propre aux actuelles théories en psychosomatique ${ }^{2}$ par une perspective "analogique". La présentation rapide d'un cas, celui de Flora, va permettre de présenter certains éléments prospectifs, concernant ce qu'il est possible d'attendre d'un tel changement de perspective, tout particulièrement dans le rapport du psychique au corporel, en cas de maladie somatique.

"Moi, on me juge à la couleur de ma peau...". C'est donc par cette phrase que se présente Flora, 25 ans, venue rencontrer un psychologue parce que l'eczéma qui recouvre certaines parties de son corps depuis l'enfance n'évolue toujours pas, oscillant indéfiniment entre des poussées spectaculaires et envahissantes, et des accalmies - voire des disparitions - plus ou moins durables. A la recherche d'une solution "globale" de son problème dermatologique, Flora me consulte en considérant - ayant lu certains de mes écrits - que je suis "spécialisé psychosomatique". Lors du premier entretien, recherchant un exemple de ces situations problématiques responsables - selon elle - de faire surgir l'eczéma, elle évoque le moment où, après leur avoir rendu visite pour le weekend, elle dit au revoir à ses parents. C'est un moment de souffrance importante pour elle, dont elle ne peut rien leur dire, se contentant selon elle, de la leur montrer: "Je réagis - eczéma, je suis écarlate, ça me brûle, c'est mon moyen d'expression". Et pour signifier qu'elle n'est pas la seule dans la famille à avoir des difficultés d'expression émotionnelle, elle affirme: "Mon père non plus il ne sait pas dire son émotion. Quand il fait une bise, il cogne!". Le privilège de l'expression "libérée" revient, toujours selon elle, à sa mère qui, lors des disputes

2. La défaillance de tout ou partie de l'appareil psychique comme cause du trouble somatique. 


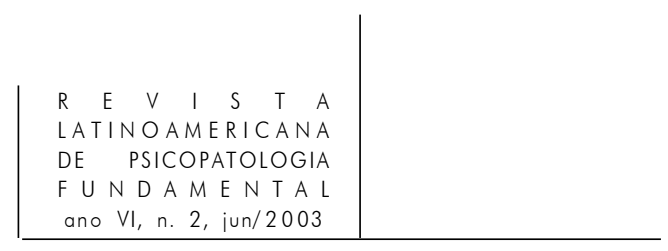

conjugales, pouvait répliquer à son mari en hurlant: “Je m'en fous que les voisins entendent!!".

Le "suivi psychologique" qui s'engage avec Flora n'aura pas, comme perspective, la guérison de l'eczéma (ce qui était son primitivement son objectif explicite), mais celle d'une tentative de "tout dire", présentée comme règle fondamentale de la démarche psychanalytique, en l'occurrence transposable à cette situation de face à face qui nous paraît, à l'un comme à l'autre, préférable à celle du divan/fauteuil.

Seul, ce changement d'objectif, énoncé comme tel, m'autorise à intégrer cliniquement ce que Fédida (1994, p. 131) appelle la "visualité figurale" des mots (celle des représentations choses inconscientes), au détriment de leur figurabilité visuelle (c'est à dire la captation imaginaire qu'ils sollicitent légitimement en médecine). Admettre, en termes de clinique psychologique, que ce sont "les mots qui observent" et non les organe de la vue, autorise dès lors l'analogisation de la pensée concernant le trouble de la peau. Il ne s'agit plus de suivre l'évolution, sur la peau, des symptômes dermatologiques, mais de repérer, dans le discours de Flora, ce que la parole l'autorise à transgresser en mêlant indistinctement psychologique et somatique.

Ainsi, au cours des deux premières années de ce suivi, Flora a été enceinte et a accouché d'une petite fille. Sur la peau du bébé et durant ses premiers mois, elle a traqué en vain l'apparition de la plus petite tache de "dessèchement" pouvant évoquer celles qui recouvrent certaines zones de sa propre peau. Son extrême précision dans la description de sa traque scopique effrénée m'incite alors à solliciter l'évocation de ce qui pourrait, dans son histoire, ressembler aux intentions qui l'accompagnaient (chercher du regard quelque chose qu'on est presque sûr de trouver). La surprise d'un souvenir, lui aussi étonnamment précis, est alors venue faire irruption dans son discours. Encore enfant, après le dîner, elle simulait régulièrement l'endormissement sur le canapé d'où ses parents regardaient la télévision. Son père se levait alors, et, la prenant délicatement "pour une fois" dans ses bras (on se souvient de sa brusquerie habituelle traduite par l'expression de Flora: "quand il fait une bise, il cogne"), l'emmenait dans sa chambre et la déposait sur son lit; sur le mur du couloir menant à la chambre, était fixé un miroir. Son plus grand plaisir était alors, en passant devant ce miroir, d'ouvrir discrètement un oeil, et de se regarder fugitivement, "lovée" dans les bras de son père. L'analogie entre ces deux traques scopiques, entreprises à de nombreuses années l'une de l'autre, a indiscutablement produit un effet de subjectivation concernant l'eczéma de Flora proprement dit.

Ses inquiétudes concernant cette pathologie se sont ainsi progressivement transformées, finissant même par s'estomper dans son discours au profit d'autres inquiétudes: des préoccupations concernant son "caractère", qu'elle incrimine 


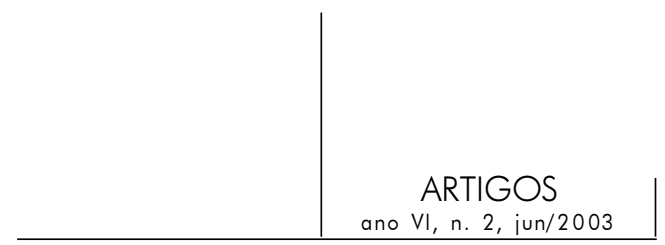

désormais à propos des difficultés relationnelles déclenchées avec son entourage - surtout depuis la naissance de sa fille -, qu'il s'agisse de son mari, de ses parents ou de sa belle famille, voire même ses collègues de travail.

Lors d'une dernière séance cependant (pour des raisons professionnelles, elle doit changer de région et interrompt donc le travail commencé avec moi), elle évoque le traitement à la ciclosporine, récemment préconisé par certains dermatologues pour le type d'eczéma qu'elle présente. Son mari, qui est cardiologue, après avoir hésité, se dit favorable à ce traitement malgré ses inconvénients, et pense qu'elle devrait l'essayer.

Voilà comment, répondant à mes sollicitations, elle se décide à en parler elle-même.

- [Comment y pensez vous, vous-même?]

- (Elle met d'abord en avant le contexte d'une dispute avec son mari) "J'ai fini par lui dire ce que j'avais sur le cour... et dans les heures qui ont suivi, j'ai eu moins d'eczéma... et puis j'ai eu une semaine sans eczéma (...) ça m'a fait du bien de dénouer tout ça avec mon mari. Je me suis dit qu'on devrait pas rester sans se parler... Moi je parle, mais pas lui. Comme il en parle peu... au sujet de la ciclosporine, je l'ai titillé... Il m'a dit qu'à son avis, enfin, à MoN avis à moi, la partie psychologique, ça représente la grosse majorité et le médicament qui permettra de le faire partir... D’ailleurs lui m'a dit que la partie psychologique était pas la plus importante... il a peut être pas tort... Depuis des années je fais en sourdine avec le traitement médical... et je fais fonctionner la tête à $170 \%$... Il m'a dit des trucs... 'Keller, c'est bien, mais où tu vas comme ça?'. Il le sait que quand je sors d'ici je suis bien. On a jamais discuté de l'évolution des choses... (...) Moi, la ciclosporine... je fais mon petit bonhomme de chemin sans ça... Mais peut être que là, faudrait que j'écoute un peu... (...) il fait des compliments que je crois pas... il me félicite pour mon travail, il en est fier! Si j'assimilais le compliment comme le reproche et l'agression, ça irait mieux! l'agression, ça pénètre!

- ["L'agression, ça pénètre" ? ?]

- Ca, c'est une bonne image! ! la dermato qui me critique, ça m'atteint jusque à la pointe des cheveux! (...) Avec le traitement médical que j'ai, je sens le mal être... l'eczéma... Mais si j'ai un traitement médical qui marque, comment je vais travailler sur ce qui me fait avoir cet eczéma? C'est difficile à expliquer à un médecin ça: “... je préfère garder encore un peu mon eczéma...”. L’idée de me soigner pour me soigner, je ne suis pas persuadée que ce soit la meilleure des choses... qu'en pensez vous?

- [Ma réponse - non reproduite ici - s'appuie sur l'idée qu'il est nécessaire de faire entendre son "savoir" sur son eczéma, sans pour autant ignorer les connaissances de la médecine sur cette maladie de peau] 
__ "On me laisse pas le temps... Les médecins... c’est médical!".

Le discours de Flora apparaît comme une tentative sans cesse renouvelée, visant à placer sur un même plan altérations corporelles et avatars psychiques. Mon engagement clinique auprès d'elle, bien qu'arrimé à l'hypothèse de l'inconscient, implique également, en référence au travail d'analogisation, de susciter avec elle un travail de la dissemblance; ce travail permet, on le constate, de respecter toutefois les liens qui se renouent continuellement dans son discours, entre les deux registres de l'organique et du psychique.

On peut observer par ailleurs qu'un tel travail psychique, indissociable d'une mobilisation transférentielle, a permis à Flora de se faire entendre autour d'elle, y compris par l'intermédiaire de l'investissement subjectif de son eczéma. Enfin, seul le "suivi" à long terme de Flora aura permis de mettre en évidence que, si le schéma - classique - de poussées eczémateuses liées à un "défaut" d'expression émotionnelle se maintient dans son discours, ce schéma va progressivement se doubler d'un rattachement à la généalogie familiale, évocateur de ce que Gori et Del Volgo appellent le "roman de la maladie".

Sans développer plus avant cet exemple d'un "instant de dire", et pour conclure la réflexion que je propose sur le travail d'analogisation dans un contexte de maladie somatique, je soulignerai à quel point il rejoint, sans s'y fondre, le travail analytique décrit par Del Volgo comme "(mettant) en évidence que toute expérience sensorielle et émotionnelle désagréable peut s'inscrire dans une conception où les traces mnésiques refoulées et le corps sexuel infantile s'emparent de ces sensations pour dire ce qui, de l'histoire du sujet, est resté en souffrance". Quant à l'éthique de la méthode analytique mobilisée pour l'occasion, elle permet de rappeler à quel point, dans ces conditions, "la recherche du vrai prévaut sur le souci du bien" (Del Volgo, 1997, p. 219).

\section{References}

Amiel-Lebigre, F. et Gognalons-Nicolet, M. Entre santé et maladie. Paris: P.U.F., 1993.

Bruchon-Schweitzer, M. et. Dantzer, R. Introduction à la psychologie de la santé. Paris: P.U.F., 1994.

Del Volgo, M.-J. L'instant de dire. Paris: Erès, 1997.

Ducousso-Lacaze, A. et Keller, P-H. Maladie, métaphore et analogie: une approche clinique et théorique, Revue Internationale de Psychopathologie, n. 20/95, p. 61540, P.U.F., 1995. 


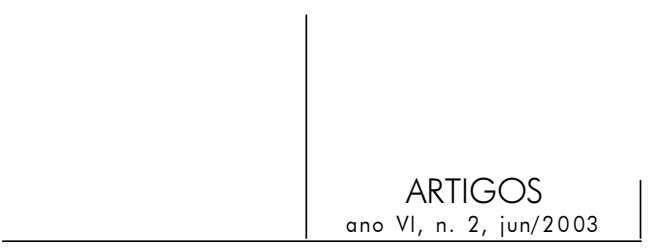

DURIF-BRUCKERT, C. Une fabuleuse machine; Anthropologie des savoirs ordinaires sur les fonctions physiologiques. Paris: Seuil, 1994.

FÉdidA, P. Pour une métapsychologie analogique. Fécondité de l'hétérogène. In: Somatisation, psychanalyse et sciences du vivant. Paris: ESHEL, 1994.

Ferenczi S., 1932, La confusion de langues entre les adultes et l'enfant. In: Psychanalyse IV, Euvres complètes, 1927-1933. Paris: Payot.

Gori, R. et Del Volgo, M.-J. L'agir psychosomatique. Cliniques méditerranéennes, n. 31/32, CIRPC, Aix-en-Provence, 1991.

Herzlich, C. \& Pierret, J. Malades d'hier, malades d'aujourd'hui. Paris: Payot, 1990.

KELLER, P-H. La médecine psychosomatique en question ou Le savoir du malade. Paris: Odile Jacob, 1997.

Médecine et psychosomatique. Paris: Flammarion, 2000.

Soin physique, soin psychique: l'analogie dans le soin. In: Qu'est-ce-que soigner? Paris: La Découverte-Syros, 2000, p. 172-90.

Propos sur les troubles dits "psychosomatiques", Revista Latinoamericana

Psicopatologia Fundamental, v. V, n. 4, décembre 2002.

Laplantine, F. Anthropologie de la maladie. Paris: Payot, 1986.

Lebreton, D. Corps et société, Librairie des Méridiens, Klincksieck et C Ce, 1985.

Secrétan, Ph.. L'analogie,. Paris: P.U.F., 1984.

Resumos

As teorias em psicossomática foram elaboradas a partir da confrontação entre medicina e psicanálise. Apesar dos progressos da medicina tecnocientífica, os estudos atuais da psicanálise efetivados no contexto dessa confrontação fecunda oferece regularmente elementos novos para a reflexão. A pesquisa apresentada aqui propõe acrescentar a "o instante de dizer" noção desenvolvida por M.-J. Del Volgo, a noção de "analogização" baseada sobre essa figura do discurso que constitui a analogia. A apresentação do caso de uma paciente sofrendo de eczema permite dar a sua plena consistência para essa noção, cujos efeitos podem, no final, renovar a visão defectologista própria à corrente psicossomática.

Palavras-chave: Psicossomática, psicanálise, analogia, eczema

Las teorías en psicosomática fueron elaboradas por confronto del psicoanálisis con la medicina. A pesar de los progresos de la medicina técnico-científica, los trabajos actuales en psicoanálisis, realizados en el contexto de esa fecunda confrontación, aportan regularmente nuevos elementos de reflexión. La búsqueda que aquí se presenta, se propone acrecentar al "instante de decir", noción desarrollada por M.-J. Del Volgo, la noción de "analogización”, fundada en el análisis de la figura del discurso que es la analogía. 


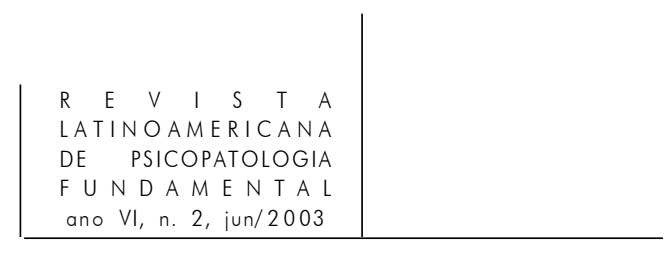

La presentación del caso de una paciente afectada por un eczema permitirá dar toda su consistencia a esa noción cuyos efectos pueden finalmente renovar la visión "defectológica" propia de la corriente llamada "psicosomática".

Palabras clave: Psicosomática, psicoanálisis, analogía, eczema

Theories in psychosomatics have been drawn up in the confrontation between psychoanalysis and medicine. In spite of the advances in techno-scientific medicine, present-day psychoanalysis, carried out in the context of this fertile confrontation, often brings up new aspects for consideration. The present article adds to the "instant of saying," a notion developed by M-J. Del Volgo, the notion of "analogization," founded on the analysis of analogy as a figure of speech. The presentation of the case of a patient suffering from eczema gives fuller consistency to this notion, the effects of which may in fact renew the "defectological" perspective proper to the current known as "psychosomatic."

Key words: Psychosomatics, psychoanalysis, analogy, eczema 